\title{
OPEN Differentiation of human adult-derived stem cells towards a neural lineage involves a dedifferentiation event prior to differentiation to neural phenotypes
}

\author{
Carlos Bueno ${ }^{1 凶}$, Marta Martínez-Morga², David García-Bernal' ${ }^{3}$, José M. Moraleda ${ }^{3}$ \& \\ Salvador Martínez ${ }^{1}$
}

\begin{abstract}
Although it has been reported that mesenchymal stem cells isolated from adult tissues can be induced to overcome their mesenchymal fate and transdifferentiate into neural cells, the findings and their interpretation have been challenged. The main argument against this process is that the cells rapidly adopt neuron-like morphologies through retraction of the cytoplasm rather than active neurite extension. In this study, we examined the sequence of biological events during neural differentiation of human periodontal ligament-derived stem cells (hPDLSCs), human bone marrow-derived stem cells (hBMSCs) and human dental pulp-derived stem cells (hDPSCs) by time-lapse microscopy. We have demonstrated that hPDLSCs, hBMSCs and hDPSCs can directly differentiate into neuron-like cells without passing through a mitotic stage and that they shrink dramatically and change their morphology to that of neuron-like cells through active neurite extension. Furthermore, we observed micronuclei movement and transient cell nuclei lobulation concurrent to in vitro neurogenesis from hBMSCs and hDPSCs. Our results demonstrate that the differentiation of hPDLSCs, hBMSCs and hDPSCs towards a neural lineage occurs through a dedifferentiation step followed by differentiation to neural phenotypes, and therefore we definitively confirm that the rapid acquisition of the neural phenotype is via a differentiation trait.
\end{abstract}

In the classical view of cell development, embryonic stem cells differentiate into progressively more precursor cells with an increasingly restricted lineage potential, until the final mature, specialised cell types are generated and functionally integrated into their respective tissues. The differentiated state of a cell was believed to be terminal and irreversible $e^{1,2}$. While asymmetric cell division is considered to be the mechanism by which the asymmetric inheritance of cellular components during mitosis defines the distinct fate of each daughter cell ${ }^{3}$.

However, there is increasing evidence that the rules of irreversibility and germ-layer restriction can be broken ${ }^{4}$. It has long been accepted that cells can change their identity, both in vitro and in vivo, a phenomenon known as cellular plasticity ${ }^{5,6}$. Cellular conversion falls into two major categories: dedifferentiation and transdifferentiation ${ }^{4-8}$. A definitive, well-accepted criterion for dedifferentiation and transdifferentiation has yet to be established ${ }^{9}$. In its simplest form, dedifferentiation is the conversion from one differentiated cell stage to a less differentiated stage. Transdifferentiation is the conversion of one differentiated cell type into another. It has been suggested that transdifferentiation may involve a dedifferentiation step before cells differentiate to a new mature phenotype, or it may occur directly in a process that bypasses such immature phenotypes ${ }^{10}$.

${ }^{1}$ Instituto de Neurociencias de Alicante (UMH-CSIC), Campus de San Juan, 03550 Alicante, Spain. ${ }^{2}$ Department of Human Anatomy and Institute of Biomedical Research (IMIB), Faculty of Medicine, University of Murcia, 30800 Murcia, Spain. ${ }^{3}$ Internal Medicine Department and Hematopoietic Transplant and Cellular Therapy Unit, Institute of Biomedical Research (IMIB), Faculty of Medicine, University of Murcia, 30800 Murcia, Spain. ${ }^{\square}$ email: cbueno@umh.es 
Literature published over the past 20 years have shown that mesenchymal stem cells (MSCs) isolated from adult tissues can be induced to overcome their mesenchymal fate and transdifferentiate into neural cells ${ }^{11-18}$. Although the transdifferentiation of human MSCs into neurons has aroused considerable interest, as it would have immense clinical potential in cell replacement therapy and regenerative medicine $e^{19,20}$, the findings and their interpretation have been challenged ${ }^{21,22}$. The main argument against this process is that the cells rapidly adopt neuron-like morphologies through retraction of the cytoplasm rather than by active neurite extensión ${ }^{23,24}$.

In a recent study ${ }^{25}$, we showed that human periodontal ligament-derived stem cells (hPDLSCs) can directly differentiate into neuron-like cells without passing through any mitotic stages. When hPDLSCs were exposed to a neural induction medium, we found that they rapidly underwent a dramatic change in shape and size; they initially adopted highly irregular forms before gradually contracting into round cells (neurogenesis). These round cells then slowly adopted a complex morphology and finally gave rise to a variety of neuron-like morphologies (neuronal polarisation). Furthermore, we also reported nuclear remodelling concurrent to in vitro neurogenesis from hPDLSCs.

The present study is designed to confirm that human stem cells isolated from adult tissues in the early stages of exposure to a neural induction medium adopt a neuron-like morphology because of a differentiation trait rather than an artefact. We performed time-lapse phase-contrast microscopy to record the changes in the cell morphology of hPDLSCs, human bone marrow-derived stem cells (hBMSCs) and human dental pulp-derived stem cells (hDPSCs).

We found that hPDLSCs, hBMSCs and hDPSCs can directly differentiate into neuron-like cells without passing through a mitotic stage. All three types of stem cell shrunk dramatically and changed their morphology to that of neuron-like cells with active neurite extension. Additionally, we may have evidenced micronuclei movement and transient cell nuclei lobulation in parallel with in vitro neurogenesis from hBMSCs and hDPSCs. Our results clearly indicate that the differentiation of hPDLSCs, hBMSCs and hDPSCs towards a neural lineage occurs through a dedifferentiation step before the cells differentiate to neural phenotypes. These findings definitively establish that the rapid acquisition of a neuron-like morphology during neural differentiation is due to a differentiation trait as opposed to an artefact.

\section{Results}

Time-lapse microscopy of hPDLSCs cultured in neural induction media. To conclusively affirm that hPDLSCs adopt a neuron-like morphology in the early stages of exposure to a neural induction medium is due to active neurite extension rather than retraction of the cytoplasm, we performed time-lapse phase-contrast microscopy and immunocytochemical analysis within the first $24 \mathrm{~h}$ of neural differentiation.

Time-lapse imaging revealed that, after neural induction, the hPDLSCs underwent a rapid change in shape and size, first adopting highly irregular forms and then gradually contracting into round cells. Subsequently, we observed the growth of new neurites from the cell body of round cells (Fig. 1, black arrows). The round cells were also noted to gradually adopt a complex morphology, acquiring dendrite-like (Fig. 2a, white arrows) and axon-like identities (Fig. 2a, red arrows), giving rise to a variety of neuron-like morphologies (Figs. 1, 2).

In addition, cytoskeletal protein $\beta$-III tubulin and F-actin staining showed that hPDLSC-derived neuron-like cells developed distinct dendrite-like and axon-like domains (Fig. 2b). Time-lapse imaging revealed that the hPDLSC-derived neuron-like cells connected to one another through different types of interactions (Fig. 2c, red arrows), including axodendritic-like contacts (Fig. 2c, yellow arrows).

hPDLSCs did not differentiate at the same time, so the cell culture simultaneously contained hPDLSCs at different stages of neurogenesis and neuronal polarisation. The results demonstrate that hPDLSCs can directly differentiate into neuron-like cells without passing through a mitotic stage, thus definitively confirming that the rapid acquisition of a neuron-like morphology during neural differentiation is by means of a differentiation trait rather than merely an artefact.

Time-lapse microscopy of hBMSCs and hDPSCs cultured in neural induction media. Isolation, characterisation and neural differentiation of multipotent stem cells from human bone marrow ${ }^{11,19,20,26,27}$ and dental pulp ${ }^{28-31}$ have been described previously. Under proliferation conditions, hBMSCs and hDPSCs displayed a fibroblast-like morphology with actin microfilaments and $\beta$-III tubulin microtubules oriented parallel to the cell's longitudinal axis (Fig. S1). During interphase, undifferentiated hBMSCs and hDPSCs had an ellipsoidal nucleus, often located in the centre of the cell (Fig. S1). These results are consistent with previous studies that found hBMSCs and dental-derived stem cells exhibited spontaneous expression of neural marker $\beta$-III tubulin even without neural induction ${ }^{32}$.

To determine whether the same rapid morphological changes could also be induced in other human adultderived stem cells, we tested hBMSCs and hDPSCs under the same neural induction conditions. As with hPDLSCs, time-lapse imaging revealed that after neural induction hBMSCs and hDPSCs underwent a dramatic change in shape and size, first adopting highly irregular forms, before gradually contracting into round cells. Subsequently, we noted the growth of new neurites from the cell body of hBMSC-derived (Fig. 3a, black arrows) and hDPSC-derived round cells (Fig. 3b, black arrows).

We also observed that hBMSC-derived (Fig. 4a) and hDPSC-derived neuron-like cells (Fig. 4b) gradually adopted a complex morphology, acquiring dendrite-like (Fig. 4, white arrows) and axon-like identities (Fig. 4, red arrows), and giving rise to a variety of neuron-like morphologies (Figs. 3, 4, 5). Cytoskeletal protein $\beta$-III tubulin and F-actin staining showed that hBMSC-derived (Fig. 5a) and hDPSC-derived neuron-like cells (Fig. 5b) developed distinct dendrite-like and axon-like domains. Morphological analysis also revealed that hBMSCderived neuron-like cells were interconnected by different types of interactions, including dendrodendritic-like contacts (Fig. 5c, red arrows) and axoaxonic-like contacts (Fig. 5c, yellow arrows). 

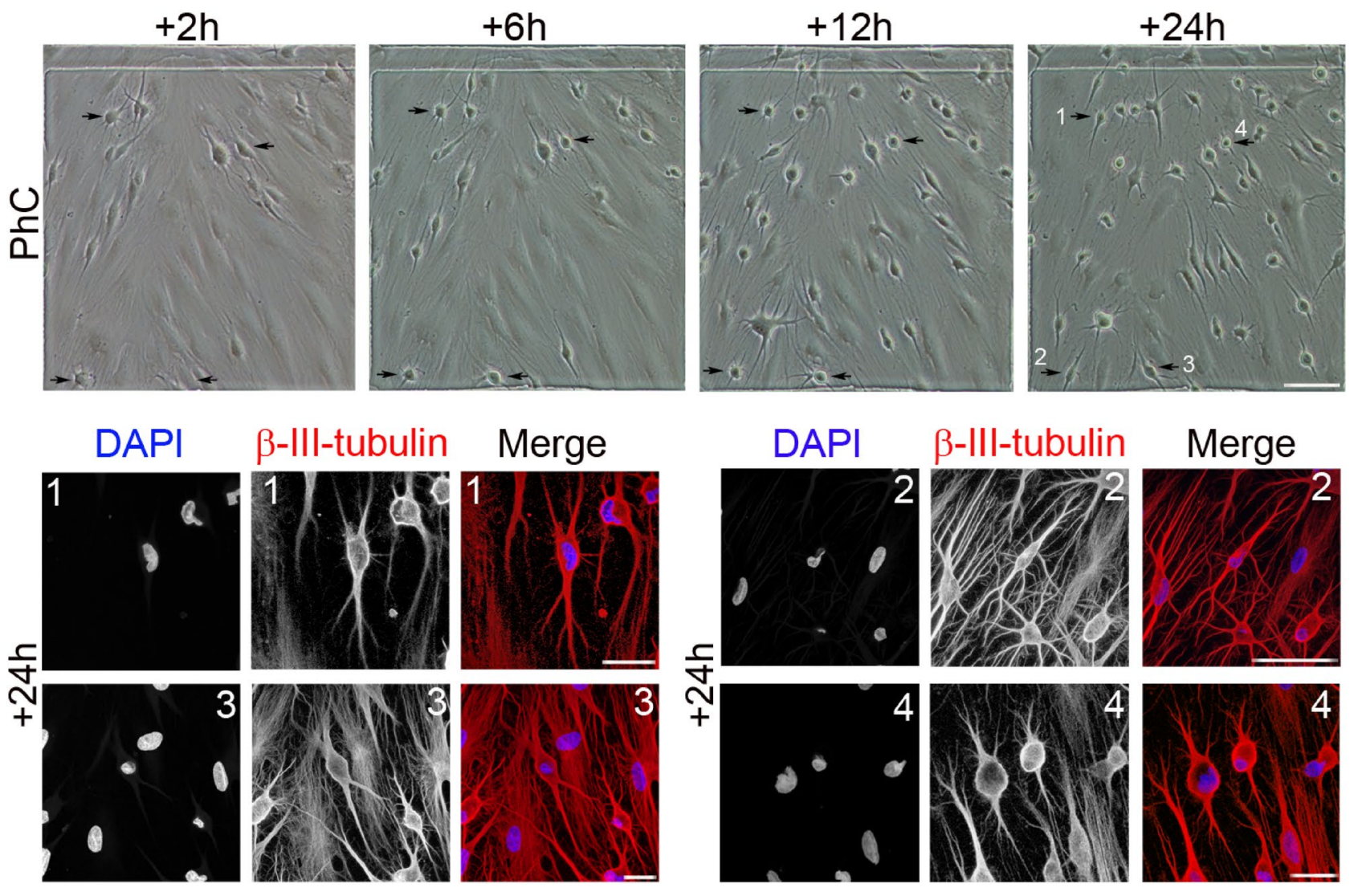

Figure 1. Morphological changes in hPDLSC cultures during neural induction. Time-lapse imaging and immunocytochemical analysis revealed that, after neural induction, hPDLSCs underwent a rapid change in shape and size, first adopting highly irregular forms and then gradually contracting into round cells. Subsequently, there was growth of new neurites from the cell body of round cells (black arrows) (numbers correspond to the areas shown in higher magnifications). The scale bars are $100 \mu \mathrm{m}$ in the phase-contrast photomicrographs, and $25 \mu \mathrm{m}$ for confocal micrographs. PhC: Phase-contrast photomicrographs.

The results indicate that there was no cell proliferation during neurogenesis from hBMSCs and hDPSCs. The undifferentiated spindle-shaped cells were reset and started their neuronal development as round spheres. We observed that the hBMSCs and hDPSCs did not differentiate at the same time and therefore the cell culture simultaneously contained hBMSCs and hDPSCs at different stages of neurogenesis and neuronal polarisation.

These findings indicate that hPDLSCs, hBMSCs and hDPSCs have similar morphological neural development sequences in vitro. The differentiation of hPDLSCs, hBMSCs and hDPSCs towards a neural lineage occurs through a dedifferentiation step before the cells differentiate to neural phenotypes.

Nuclear remodelling. In a recent $s t u d y^{25}$, we showed that nuclear remodelling occurred during in vitro neurogenesis from hPDLSCs. We discovered that many hPDLSCs exhibit unusual nuclear structures and extranuclear bodies in the cellular cytoplasm when hPDLSCs approach a spherical morphology (neurogenesis). In addition, no unusual nuclear structures were observed as hPDLSC-derived neuron-like cells gradually acquired a more mature neuron-like morphology (neuronal polarisation).

While we acknowledge that the definitive nuclear remodelling sequence, when hPDLSCs become nearspherical, can only be determined by detecting fluorescent labelled cell nuclei in time-lapse microscopy, our accumulated data suggest how these steps may occur ${ }^{25}$. Chromatin-containing bodies arise from the main nuclei and start to move towards specific positions within the cell, temporarily forming lobed nuclei. Subsequently, these lobed nuclei connect together to form nucleoplasmic bridges and finally the internuclear bridges facilitate the merger into a single nucleus with an eccentric position within the cell.

To determine whether nuclear remodelling also occurs when hBMSCs and hDPSCs take on a near-spherical shape, we differentiated hPDLSCs, hBMSCs and hDPSCs in the same neural induction medium (Figs. 6, 7, 8).

Within $2 \mathrm{~h}$ of exposure to the neural induction medium we observed DNA-containing structures arise from the main nuclei of hPDLSCs (Fig. 6a), hBMSCs (Fig. 7a) and hDPSCs (Fig. 8a). We also observed DNA-containing structures moving away from the main nuclei of and towards specific positions within hPDLSCs (Fig. 6b, c, respectively), hBMSCs (Fig. 7b, c) and hDPSCs (Fig. 8b, c), where they temporarily formed lobed nuclei.

Within $24 \mathrm{~h}$ of exposure to the neural induction medium we observed lobed nuclei in hDPSCs (Fig. 8d), lobed nuclei connecting together to form nucleoplasmic bridges in hDPSCs (Fig. 8e), lobed nuclei connected by internuclear bridges in hPDLSCs (Fig. 6d, red arrows), hBMSCs (Fig. 7d, red arrows) and hDPSCs (Fig. 8f), 
a
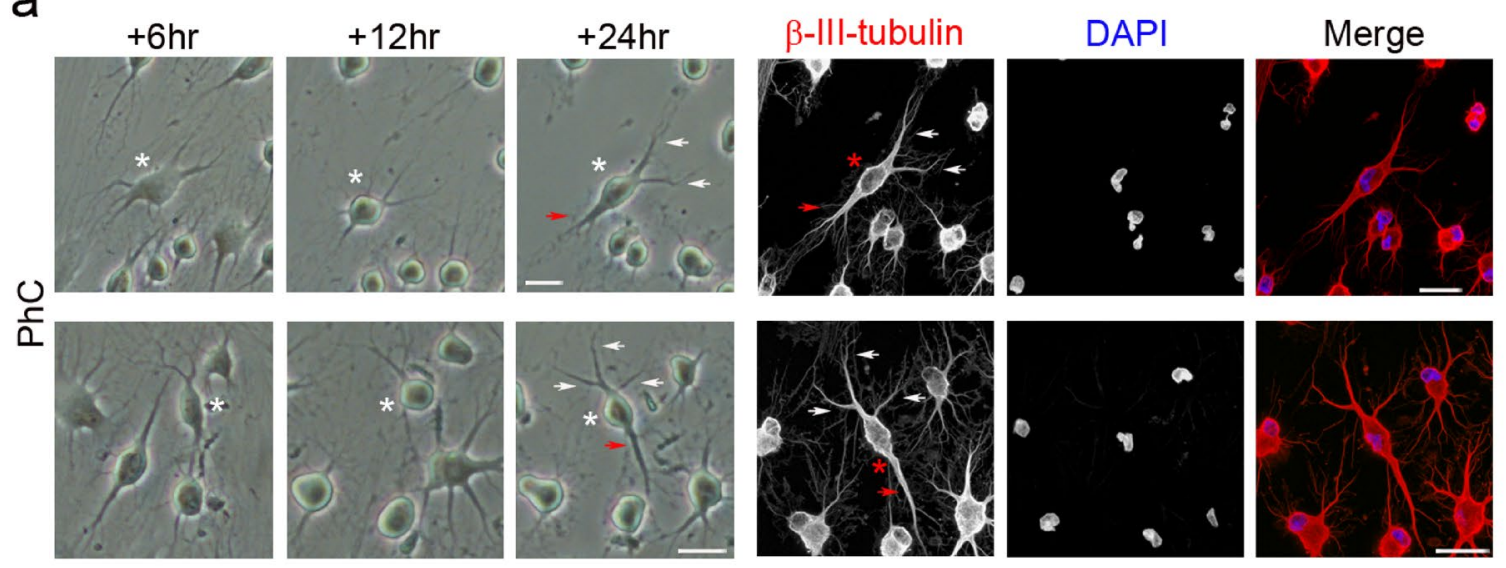

b
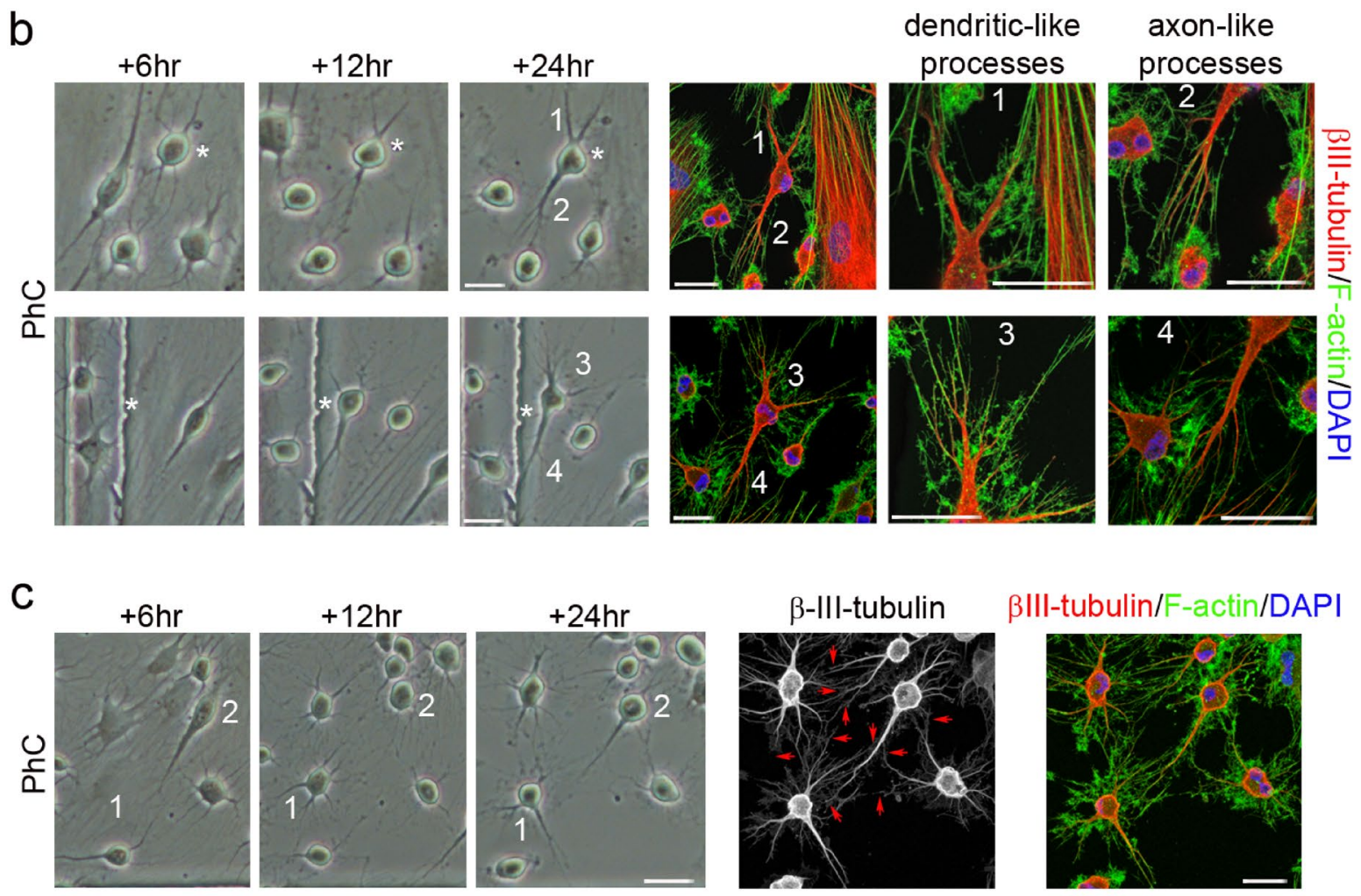

$\beta$ III-tubulin/F-actin/DAPI
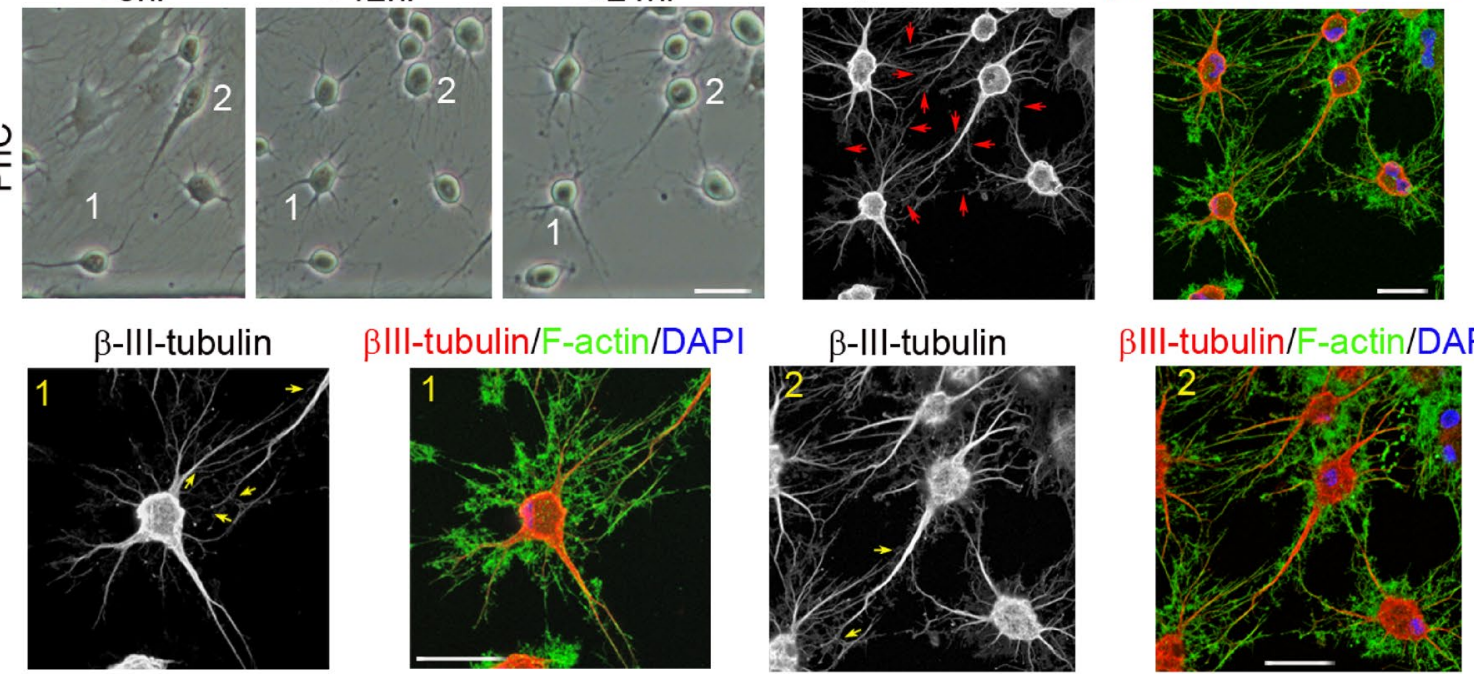

$\beta$ III-tubulin/F-actin/DAPI

Figure 2. Neuronal polarisation of hPDLSC-derived neuron-like cells. (a) Time-lapse imaging and immunocytochemical analysis revealed that round cells gradually adopted a complex morphology, acquiring dendrite-like (white arrows) and axon-like identities (red arrows). (b) Cytoskeletal protein $\beta$-III tubulin and F-actin staining showed that hPDLSC-derived neuron-like cells developed distinct dendrite-like and axonlike domains (numbers correspond to the areas shown in higher magnifications). (c) Time-lapse imaging and immunocytochemical analysis also revealed that the hPDLSC-derived neuron-like cells connected together through different types of interactions (red arrows), including axodendritic-like contacts (yellow arrows) (numbers correspond to the areas shown in higher magnifications). Scale bar: $25 \mu \mathrm{m}$. PhC: Phase-contrast photomicrographs. 
a hBMSCs
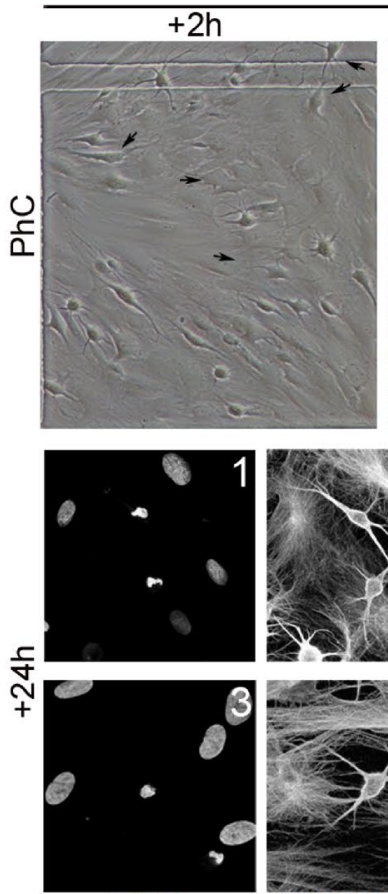

DAP

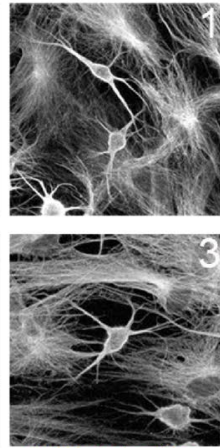

$\beta$-Ill-tubulin
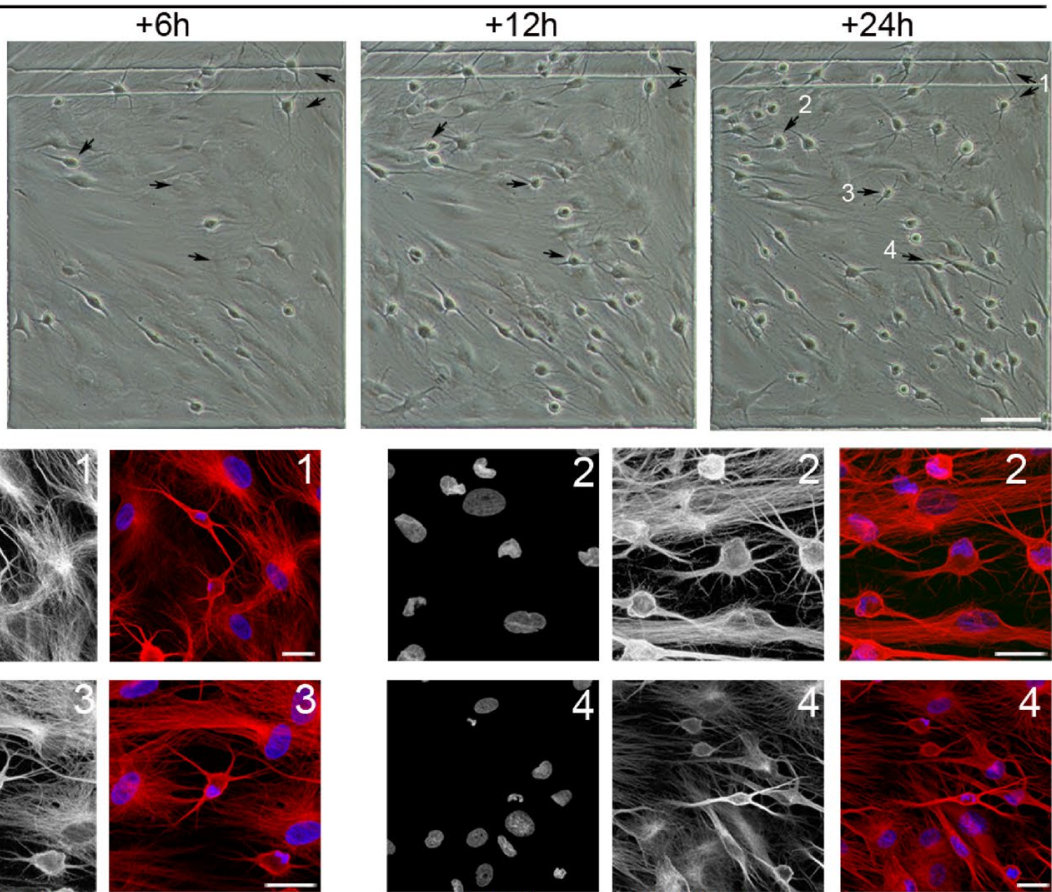

Merge
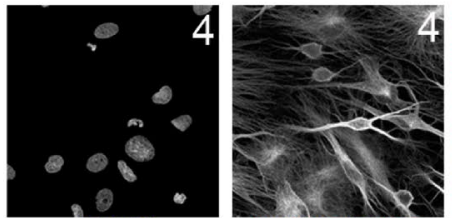

$\beta$-IIl-tubulin

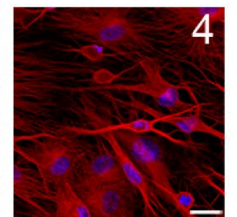

Merge

b
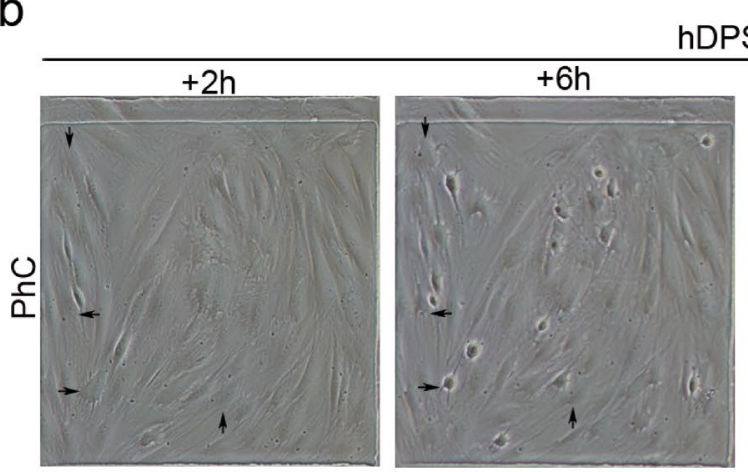

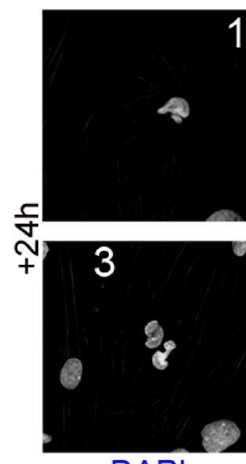

DAPI
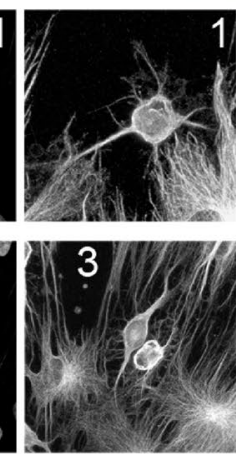

$\beta$-III-tubulin
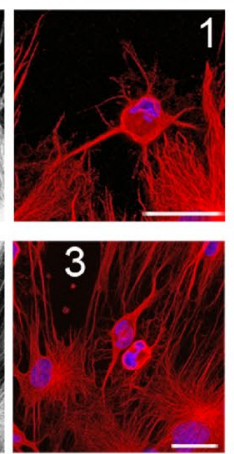

Merge

hDPSCs
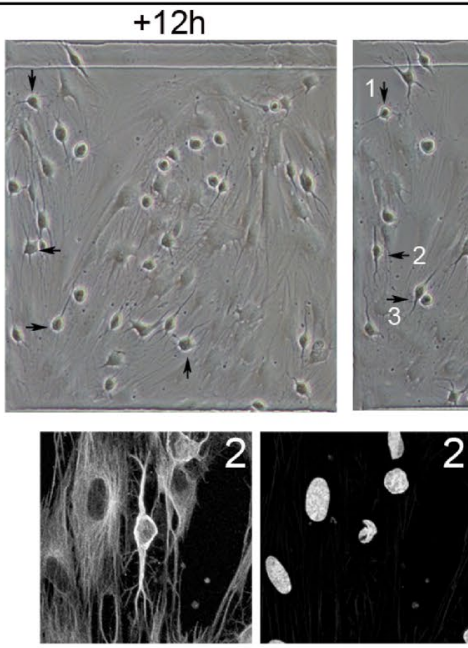

$+24 h$
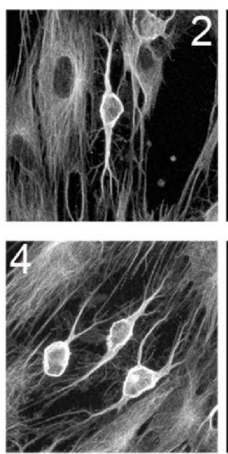

DAPI
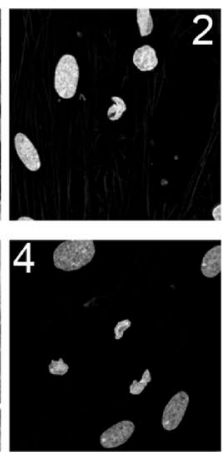

$\beta$-III-tubulin
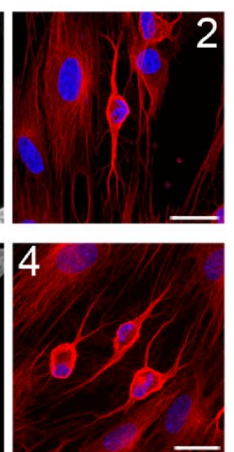

Merge

Figure 3. Morphological changes in hBMSC and hDPSC cultures during neural induction. Time-lapse imaging and immunocytochemical analysis revealed that, after neural induction, hBMSCs (a) and hDPSCs (b) underwent a rapid change in shape and size, first adopting highly irregular forms and then gradually contracting into round cells. Subsequently, there was growth of new neurites from the cell body of round cells (black arrows) (numbers correspond to the areas shown in higher magnifications). The scale bars are $100 \mu \mathrm{m}$ in the phasecontrast photomicrographs, and $25 \mu \mathrm{m}$ for confocal micrographs. PhC: Phase-contrast photomicrographs. 
a hBMSCs

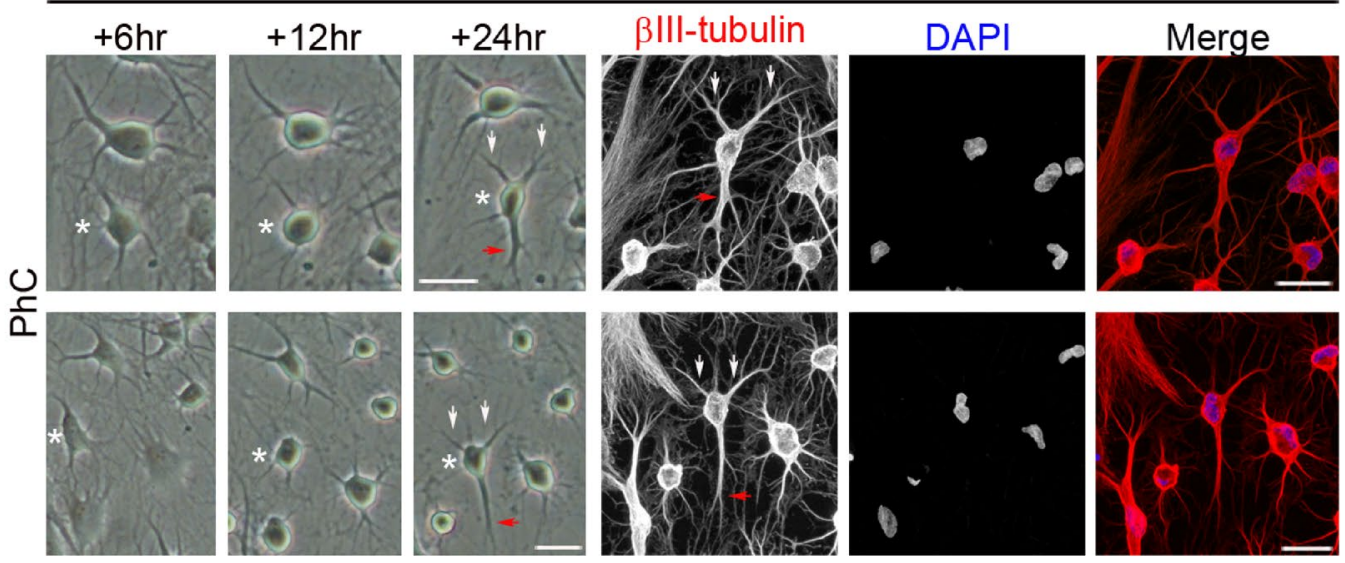

b
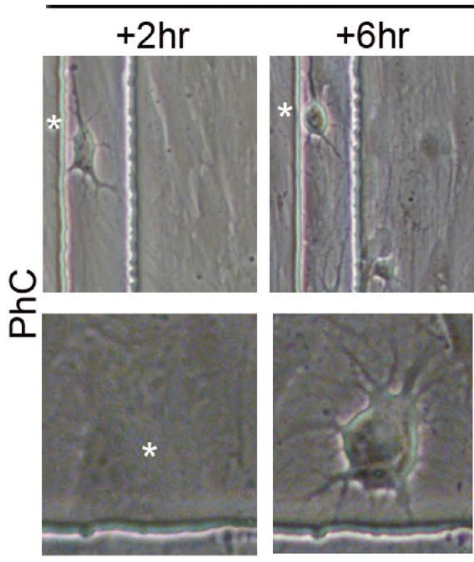

$+12 \mathrm{hr}$

hDPSCs
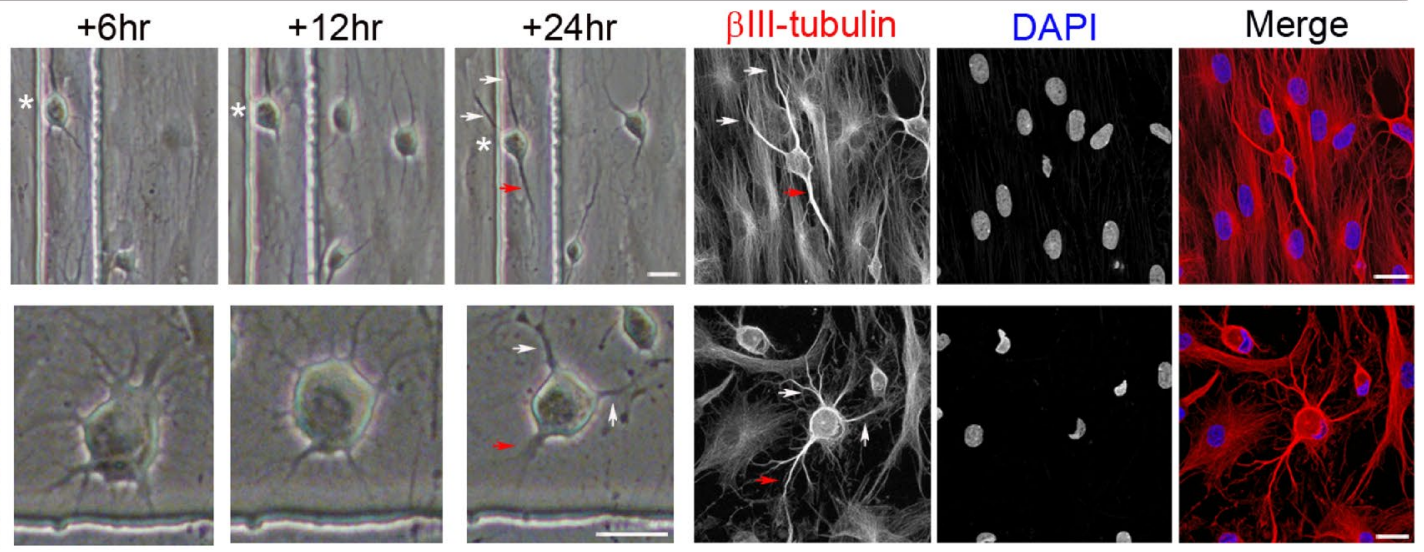

Figure 4. Early stages of neuronal polarisation of hBMSC-derived and hDPSC-derived neuron-like cells. Time-lapse imaging and immunocytochemical analysis revealed that hBMSC-derived (a) and hDPSC-derived neuron-like cells (b) gradually adopted a complex morphology, acquiring dendrite-like (white arrows) and axon-like identities (red arrows). Scale bar: $25 \mu \mathrm{m}$. PhC: Phase-contrast photomicrographs.

and lobed nuclei connected by internuclear bridges merging into a single nucleus in hPDLSCs (Fig. 6d, green arrows), hBMSCs (Fig. 7d, green arrows) and hDPSCs (Fig. 8g). No unusual nuclear structures were observed as hPDLSC-derived (Fig. 6d, yellow arrows) and hBMSC-derived neuron-like cells (Fig. 7d, yellow arrows) gradually acquired a more mature neuron-like morphology.

The results indicate that nuclear remodelling also occurred during in vitro neurogenesis from hBMSCs and hDPSCs. hBMSCs and hDPSCs also exhibited unusual nuclear structures and extranuclear bodies in the cellular cytoplasm when they approach a near-spherical shape. These DNA-containing structures displayed a spherical or ovoid shape (Fig. S2a) and some of them appeared to be connected to the main body of the nucleus by thin strands of nuclear material (Fig. S2b, yellow arrows). The hPDLSCs, hBMSCs and hDPSCs had very similar nuclear morphologies during in vitro neurogenesis. These results confirm our previous data ${ }^{25,33}$ and further support the idea that hBMSCs, hPDLSCs and hDPSCs follow similar morphological neural development sequences in vitro.

\section{Discussion}

In this study, we have shown that hPDLSCs, hBMSCs and hDPSCs can directly differentiate into neuron-like cells without passing through a mitotic stage. In fact, the spindle-shaped cells (differentiated cell stage) were reset and started their neuronal development as round spheres (less differentiated cell stage). It would, therefore, be interesting to examine whether cell rounding is a common feature of cellular plasticity.

Our results indicatate that the differentiation of hPDLSCs, hBMSCs and hDPSCs towards a neural lineage occurs through a dedifferentiation step (neurogenesis) before the cells differentiate to neural phenotypes (neuronal polarisation).

In the present work, we have demonstrated that hPDLSC-derived, hBMSC-derived and hDPSC-derived neuron-like cells produce neurites that gradually adopt a complex morphology, acquiring dendrite-like and 
a

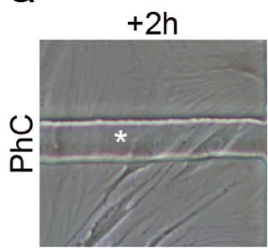

$+2 h$

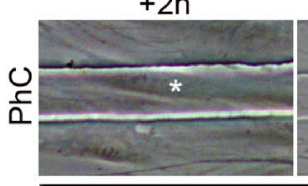

$+6 h$

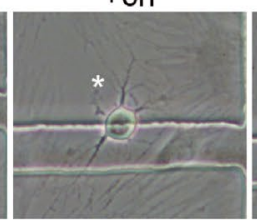

$+6 h$

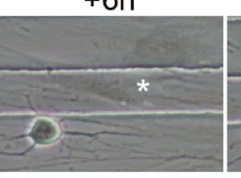

$+12 \mathrm{~h}$

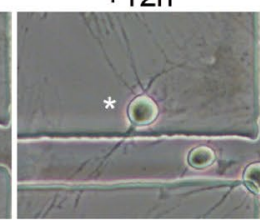

$+12 \mathrm{~h}$

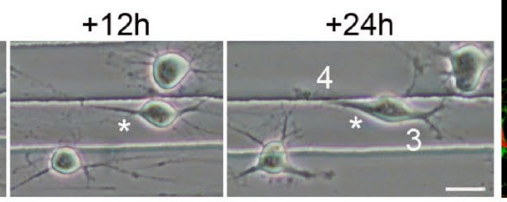

hBMSCs
$+24 h$

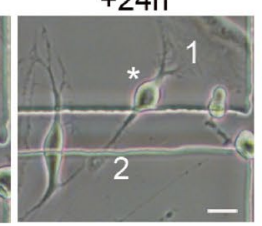

2

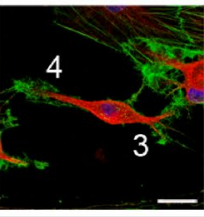

dendritic-like processes
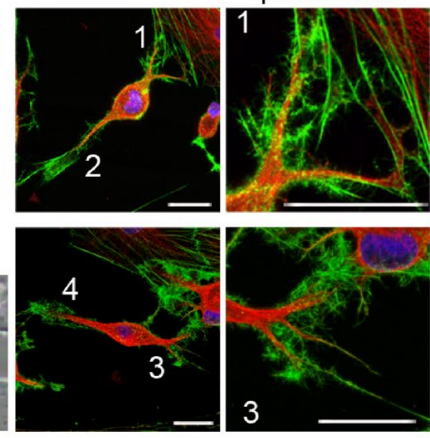

axon-like processes
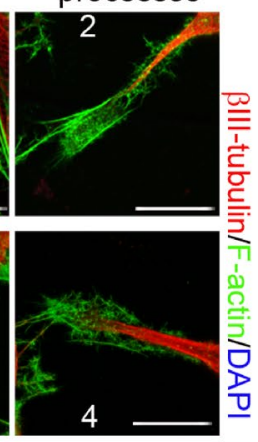

dendritic-like axon-like

b
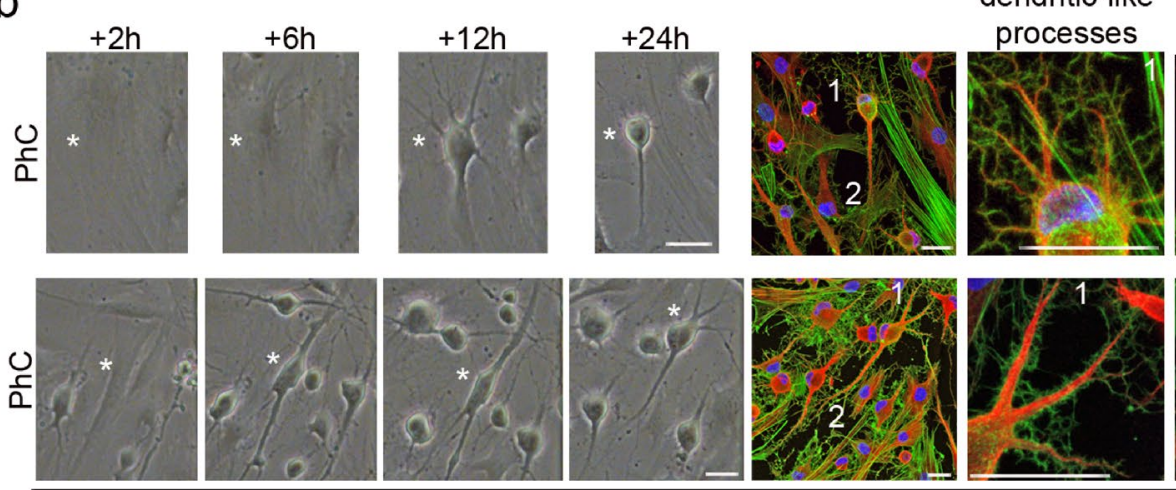

processes

hDPSCs
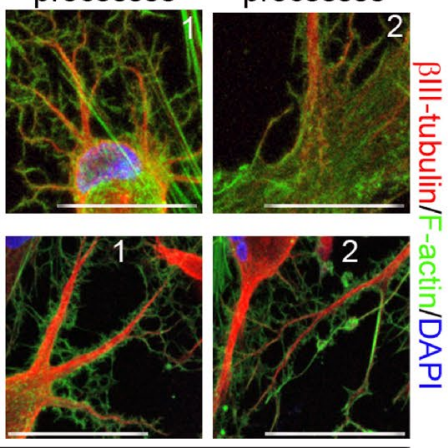

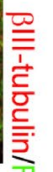

C
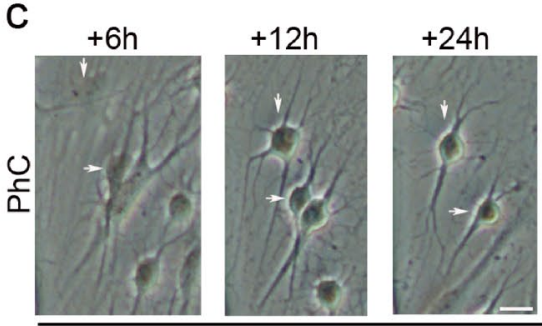

Blll-tubulin/

hBMSCs

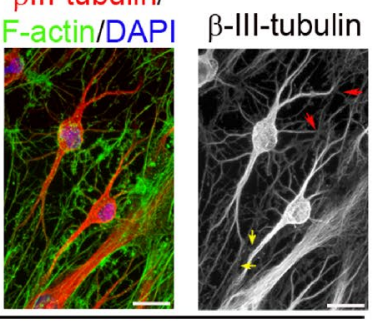

Figure 5. Final stages of neuronal polarisation of hBMSC-derived and hDPSC-derived neuron-like cells. Cytoskeletal protein $\beta$-III tubulin and F-actin staining showed that hBMSC-derived (a) and hDPSC-derived neuron-like cells (b) developed distinct dendrite-like and axon-like domains (numbers correspond to the areas shown in higher magnifications). (c) Time-lapse imaging and immunocytochemical analysis also revealed that the hBMSC-derived neuron-like cells connected together through different types of interactions, including dendrodendritic-like contacts (red arrows) and axoaxonic-like contacts (yellow arrows). Scale bar: $25 \mu \mathrm{m}$. PhC: Phase-contrast photomicrographs.

axon-like identities. Therefore, our results definitively establish that the rapid acquisition of a neuron-like morphology during neural differentiation occurs though a differentiation trait and is not just an artefact.

Although further research is required to confirm the successful differentiation of hPDLSCs, hBMSCs and hDPSCs into a neural lineage, and finally facilitate the production of autologous cells for cell replacement in a diseased central nervous system, our results provide additional evidence that cells derived from human adult tissues can be converted into neuronal cells without genetic manipulation ${ }^{34}$.

In a previous publication ${ }^{25}$, we reported that nuclear remodelling occurred during in vitro neurogenesis from hPDLSCs. We observed that many hPDLSCs had an unusual nuclear structure and chromatin-containing bodies in the cellular cytoplasm when they took on a near-spherical shape. In the present study, we have shown that nuclear remodelling also occurred during in vitro neurogenesis from hBMSCs and hDPSCs. We observed that hBMSCs and hDPSCs exhibited unusual nuclear structures and chromatin-containing bodies in the cellular cytoplasm when they became near-spherical in shape. It is important to mention that the hPDLSCs, hBMSCs 
a

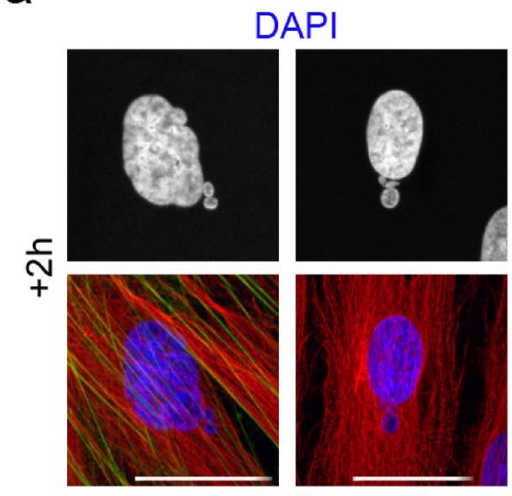

$\beta \mid I-$ tubulin/DAPI b

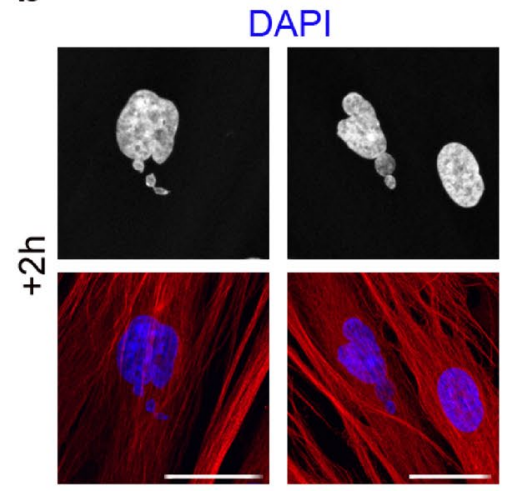

$\beta$ III-tubulin/DAPI
C

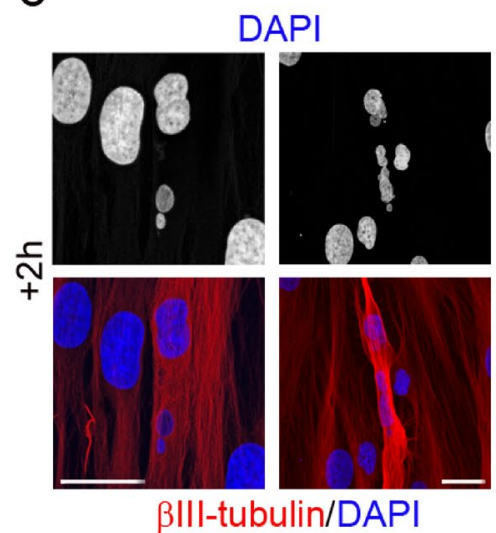

$\beta$ III-tubulin/DAPI

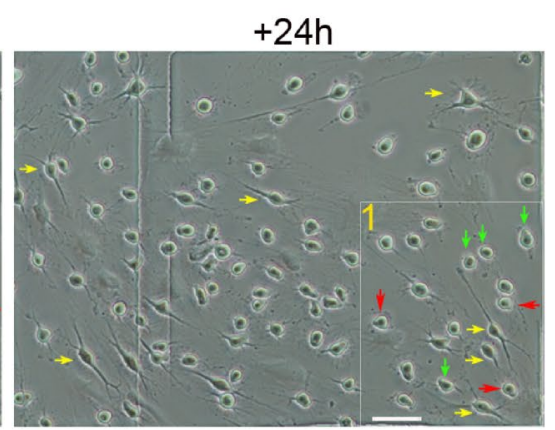

d

$+6 h$

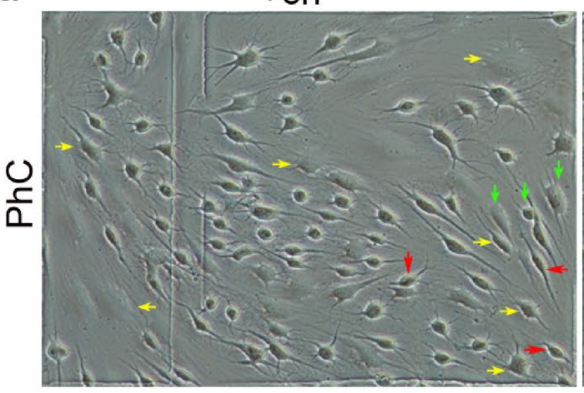

$+12 h$
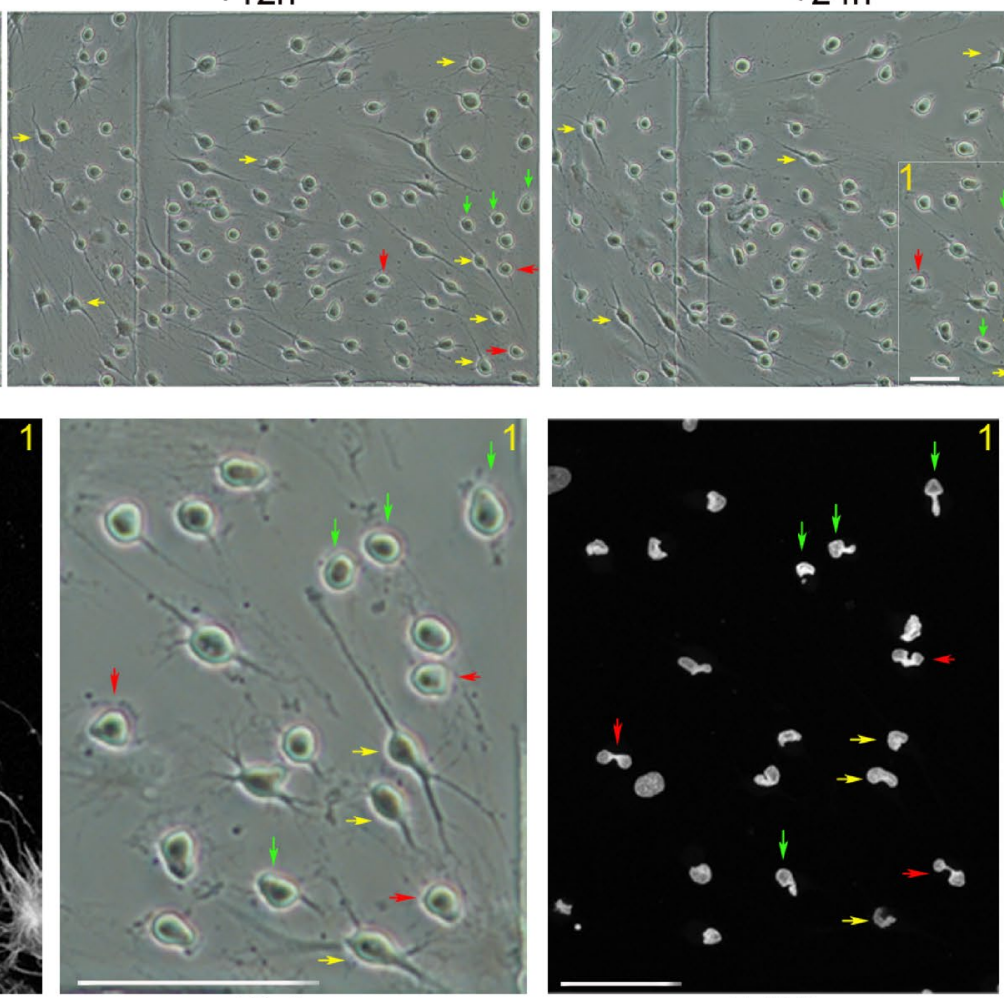

DAPI

$\beta$-III-tubulin

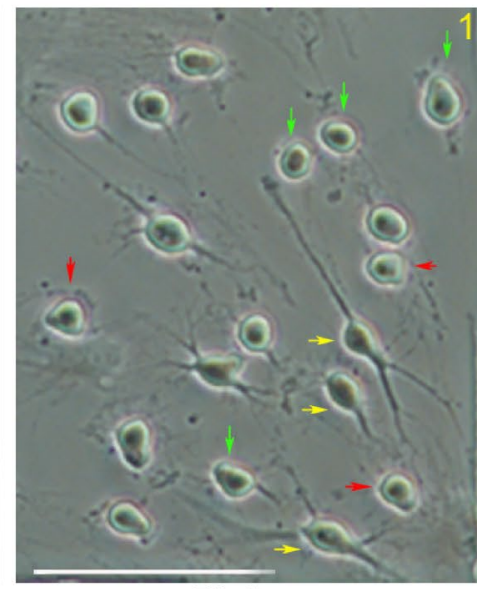

$\mathrm{PhC}$

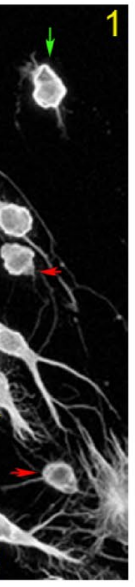

(1)

Figure 6. Nuclear shape remodelling occurs during neurogenesis from hPDLSCs. DNA-containing structures arose from the main nuclei (a) and started to move (b) towards specific positions within the cell (c), and temporarily form lobed nuclei. These lobed nuclei then connected to one another through small DNAcontaining structures to form nucleoplasmic bridges (d, red arrows). Finally, the lobed nuclei connected by internuclear bridges (d, green arrows) joined to form single nuclei with an eccentric position within hPDLSCderived neuron-like cells (d, yellow arrows). The scale bars are $50 \mu \mathrm{m}$ in the phase-contrast photomicrographs, and $25 \mu \mathrm{m}$ for confocal micrographs. PhC: Phase-contrast photomicrographs.

and hDPSCs had a very similar nuclear morphology during in vitro neurogenesis. These results confirm our previous findings ${ }^{25,33}$ and further support that hPDLSCs, hBMSCs and hDPSCs follow similar morphological neural development sequences in vitro.

These unusual nuclear structures and extranuclear bodies were described independently in mammals (including in most blood and immune cells ${ }^{35}$ ), vertebrates, invertebrates, plants and protozoa ${ }^{35-37}$. However, the exact role of these extranuclear bodies and their possible correlation with unusual nuclear structures is not yet known ${ }^{38}$. Our results suggest these unusual nuclear structures and extranuclear bodies may be associated with nuclear movement within the cell. Although future analysis involving live-cell nucleus fluorescence labelling and timelapse microscopy is necessary to determine whether chromatin-containing bodies move within the cell, and if there is any correlation between the formation of these extranuclear bodies and the unusual nuclear structures, 
a

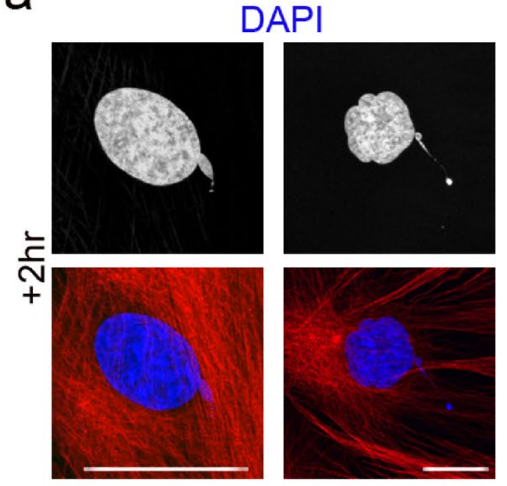

$\beta$ III-tubulin/DAPI b

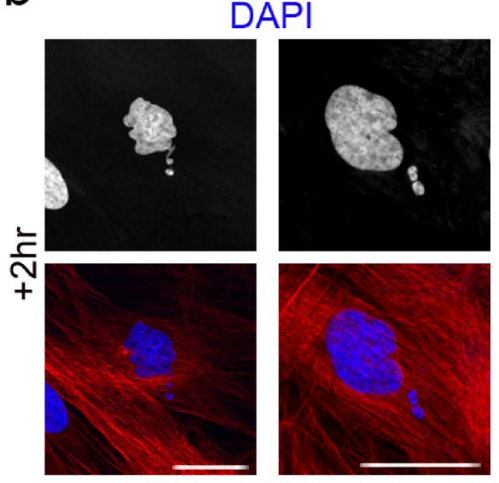

$\beta$ III-tubulin/DAPI

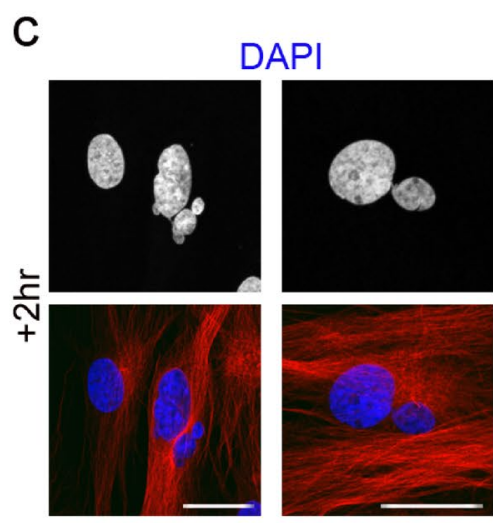

$\beta \mid I-$-tubulin/DAPI
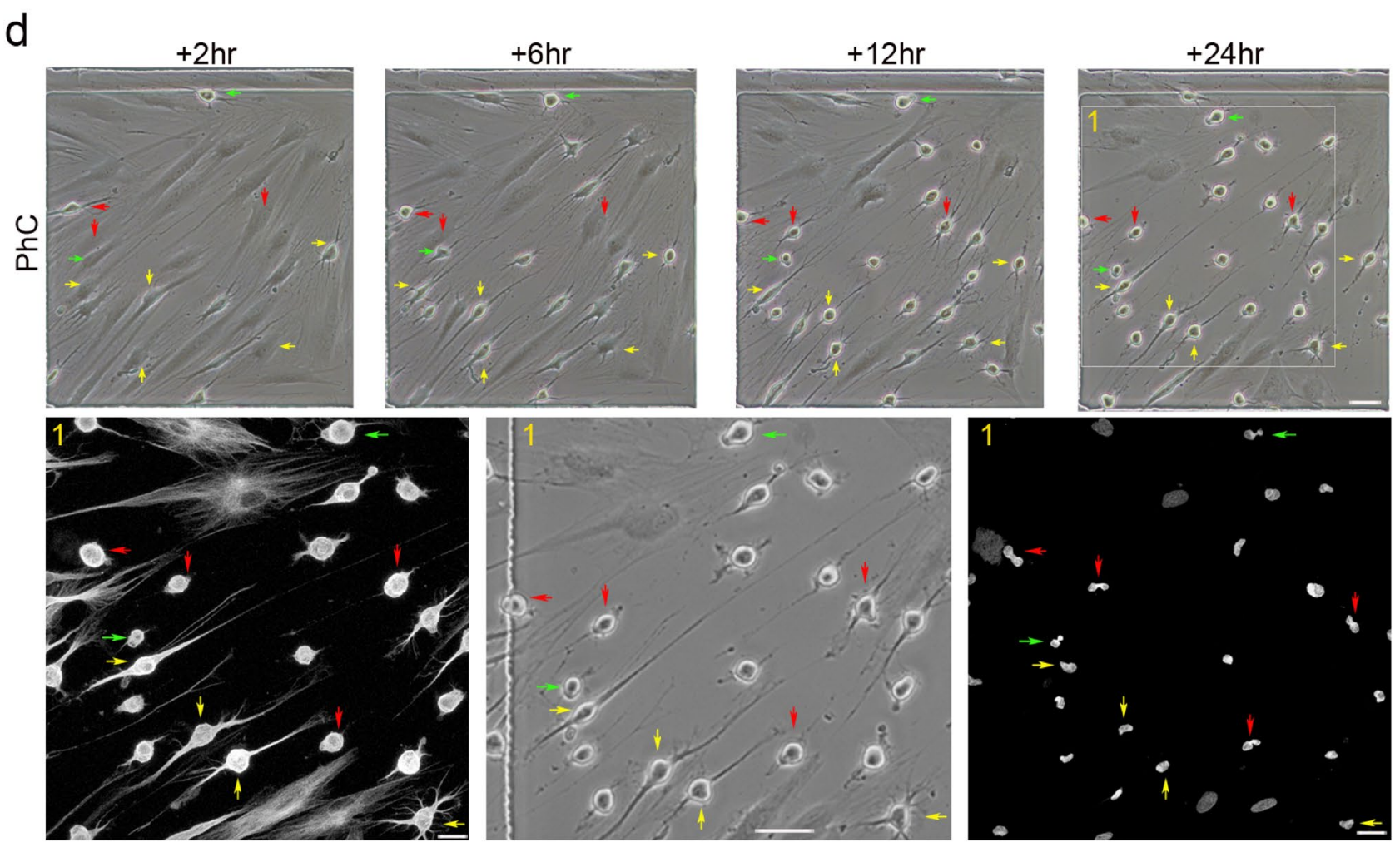

Figure 7. Nuclear shape remodelling occurs during neurogenesis from hBMSCs. DNA-containing structures arose from the main nuclei (a) and started to move (b) towards specific positions within the cell and temporarily formed lobed nuclei (c). These lobed nuclei then connected together through small DNA-containing structures to form nucleoplasmic bridges (d, red arrows). Finally, the lobed nuclei connected by internuclear bridges (d, green arrows) joined to form single nuclei with an eccentric position within hPDLSC-derived neuron-like cells (d, yellow arrows). The scale bars are $50 \mu \mathrm{m}$ in the phase-contrast photomicrographs, and $25 \mu \mathrm{m}$ for confocal micrographs. PhC: Phase-contrast photomicrographs.

a recent study has demonstrated that chromatin-containing bodies arise from the main nuclei, move within the cell and are ultimately loaded in exosomes ${ }^{39}$.

Importantly, the nuclear morphology of hPDLSCs, hBMSCs and hDPSCs observed during the dedifferentiation step (neurogenesis) bears great similarities to the nuclear morphology of neural stem cells (NSCs) located in the ventricular-subventricular zone of the anterolateral ventricle wall in the human foetal brain ${ }^{40}$ and adult mouse brain ${ }^{41-43}$; their nuclear morphology is also very similar to that of many cultured hippocampal neurons ${ }^{44}$. Although it has been suggested that these unusual nuclear structures are associated with quiescence in adult $\mathrm{NSCs}^{43}$, our results suggest that they may be associated with nuclear movement within the cell during the initial phases of neurogenesis, but without any relation to cell division.

It has generally been believed that adult neurogenesis occurs progressively through sequential phases of proliferation and neuronal differentiation of adult NSCs ${ }^{45}$. However, the current models of adult NSC maintenance 
a

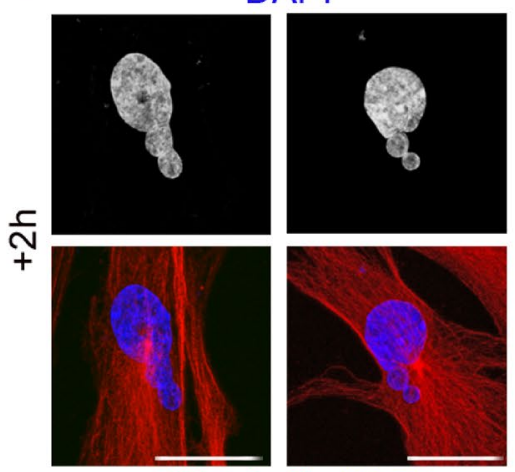

$\beta$ III-tubulin/DAPI b

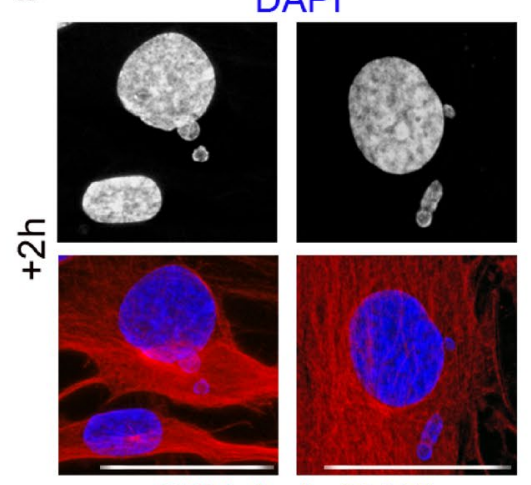

$\beta$ III-tubulin/DAPI

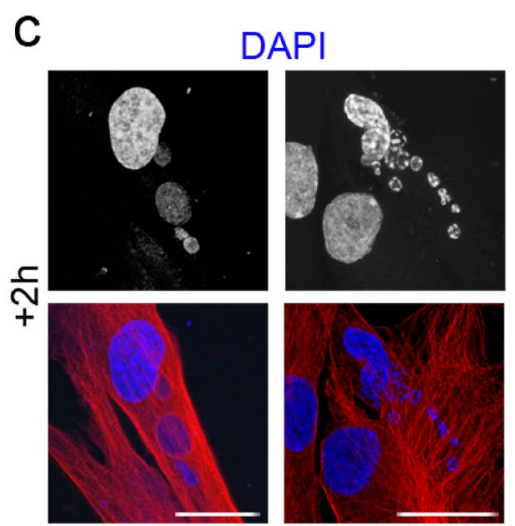

Blll-tubulin/DAPI

d
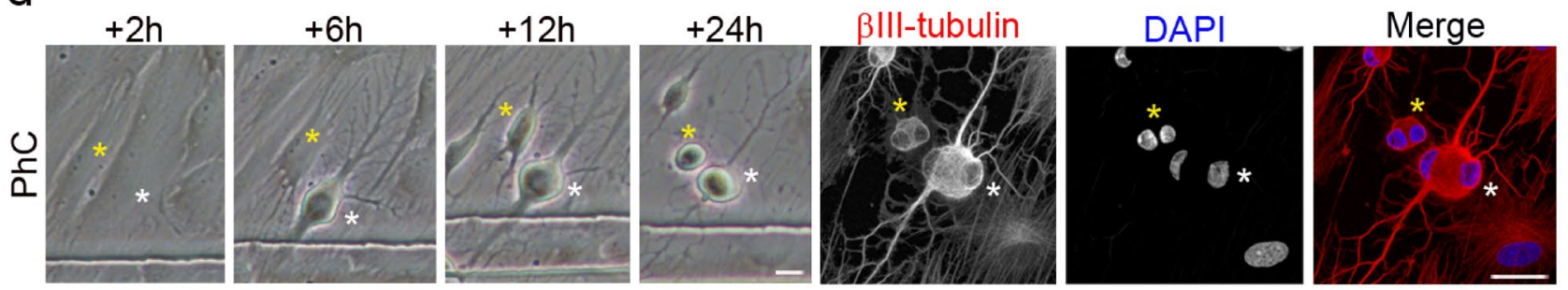

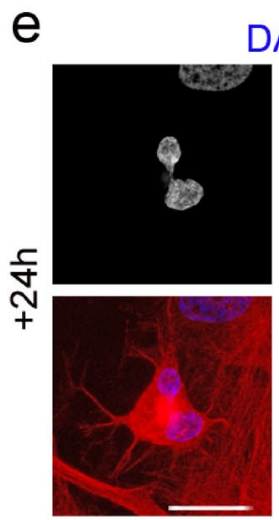

$\beta / I-$ tubulin/DAPI

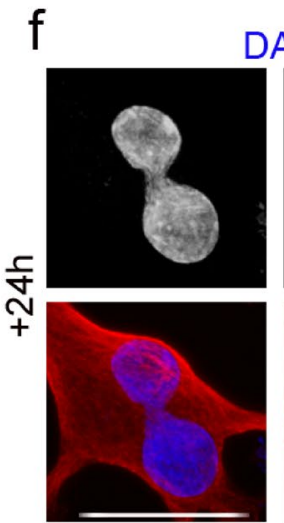

$\beta$ III-tubulin/DAPI

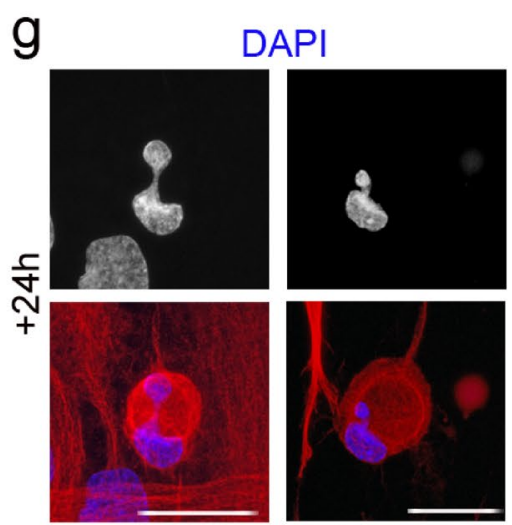

$\beta$ III-tubulin/DAPI

Figure 8. Nuclear shape remodelling occurs during neurogenesis from hDPSCs. DNA-containing structures arose from the main nuclei (a) and started to move (b) towards specific positions within the cell (c) and temporarily formed lobed nuclei (d). These lobed nuclei then connected together through small DNAcontaining structures to form nucleoplasmic bridges (e). Finally, the lobed nuclei connected by internuclear bridges (f) joined to form single nuclei with an eccentric position within hPDLSC-derived neuron-like cells (g). Scale bar: $25 \mu \mathrm{m}$. PhC: Phase-contrast photomicrographs.

and differentiation are still contentious because the approaches used to probe stem cell division and differentiation are inherently limited ${ }^{46}$.

It is important to note that very few reports describing newborn neurons in the adult brain have found mitotic chromosomes $^{47-55}$, which would confirm that adult neurogenesis occurs progressively through sequential phases of proliferation. What is more, several studies indicate that some observations interpreted as cell division could be false positives ${ }^{56-60}$. The findings mentioned above and the similarities between the nuclear morphology of hPDLSCs, hBMSCs and hDPSCs during in vitro neurogenesis compared to that of adult NSCs located in neurogenic niches and cultured hippocampal neurons suggest that adult NSCs may also convert into neurons without involving cell division. Future research is necessary to determine the likelihood of this conjecture.

As noted above, there is increasing evidence to suggest that adult cells can assume new fates without asymmetric cell division through dedifferentiation and transdifferentiation processes. These findings call into question the rules of irreversibility and germ-layer restriction as well as the classical definition of a stem cell ${ }^{4-10,61}$. 
Our results demonstrate that hPDLSCs, hBMSCs and hDPSCs retain plasticity. Therefore, these cells could also help increase our understanding of the mechanisms underlying changes in cell fate and shed light on whether differentiation, dedifferentiation and transdifferentiation occur in different contexts or are different terms for the same cellular process.

\section{Methods}

Ethical conduct of research. The authors declare that all protocols used to obtain and process all human samples were approved by the local ethics committees (UMH.IN.SM.03.16, HULP3617.05/07/2012 and HUSA19/1531.17/02/2020) according to Spanish and European legislation and conformed to the ethical guidelines of the Helsinki Declaration. Donors provided written informed consent before obtaining samples.

Isolation and culture of hPDLSCs and hDPSCs. To obtain hPDLSCs, human premolars were extracted and collected from three different healthy adult donors undergoing orthodontic therapy in Murcia dental hospital (Spain). Human periodontal ligament (hPDL) was scraped from the middle third region of the root surface. To obtain hPDSCs, human third molars were extracted and collected from three different healthy adult donors undergoing orthodontic therapy in Murcia dental hospital (Spain). Human dental pulp (hDP) was harvested from the teeth.

Dissociated cell cultures of hPDL and hDP tissues were prepared as previously described ${ }^{25,62}$. After washing the extracted hPDL and hDP with Ca and Mg-free Hank's balance salt solution (HBSS), hPDL and hDP was digested with type I collagenase $(3 \mathrm{mg} / \mathrm{ml})$ and dispase II $(4 \mathrm{mg} / \mathrm{ml})$ in alpha modification minimum essential medium eagle ( $\alpha$-MEM) for $1 \mathrm{~h}$ at $37^{\circ} \mathrm{C}$. The reaction was stopped by the addition of $\alpha$-MEM. hPDL and hDP derived from different subjects were pooled together to obtain single cell suspensions by passing the cells through a $70 \mu \mathrm{m}$ strainer (BD Falcon). Cells were centrifuged, and the pellet was resuspended in serum-containing media (designated as the basal media), composed of DMEM low glucose medium supplemented with fetal bovine serum (10\%), non-essential amino acid solution, penicillin-streptomycin (100 units/ml) and l-glutamine (2 mM). The cell suspension was plated into six-well multiwell plates (BD Falcon) and incubated at $37^{\circ} \mathrm{C}$ in $5 \% \mathrm{CO} 2$. All studies were performed using hPDLSCs and hDPSCs expanded within culture passages 3-4.

Isolation and culture of hBMSCs. Bone marrow aspirates were obtained by percutaneous direct aspiration from the iliac crest of 5 healthy volunteers at University Hospital Virgen de la Arrixaca (Murcia, Spain). Bone marrow was collected with $20 \mathrm{U} / \mathrm{ml}$ sodium heparin, followed by a Ficoll density gradient-based separation by centrifugation at $540 \mathrm{~g}$ for $20 \mathrm{~min}$. After, mononuclear cell fraction was collected, washed twice with $\mathrm{Ca}^{2+} / \mathrm{Mg}^{2+}$-free phosphate buffered saline (PBS) and seeded into $175-\mathrm{cm} 2$ culture flasks (Nunc, Thermo Fisher Scientific) at a cell density $1.5 \times 10^{5}$ cells $/ \mathrm{cm}^{2}$ in DMEM low glucose medium supplemented with fetal bovine serum (10\%), GlutaMAX (1\%) and penicillin/streptomycin (1\%). After 3 days of culture at $37^{\circ} \mathrm{C}$ and $5 \% \mathrm{CO}_{2}$, non-attached cells were removed and fresh complete medium was added. Culture media were renewed every 2 days, and the isolated hBMSCs were passaged when cultures were $70-80 \%$ confluent. All studies were performed using hBMSCs expanded within culture passages 3-4.

Time-lapse microscopy of hPDLSCs, hPDSCs and hBMSCs cultured in neural induction media. We used $\mu$-Dish $35 \mathrm{~mm}$, high Grid- 500 (Ibidi) for live cell imaging. Numeric marks on the bottom of each dish allow users to identify the location of cells. Cells were plated onto collagen IV coated plastic or glass coverslips, and maintained in basal media or neural induction media.

To induce neural differentiation, cells at passage 3-4 were allowed to adhere to the plates overnight. Media was removed the following day and the cells were cultured for 2 days in serum-free media (designated as the neural basal media) consisting in Dulbecco's modified Eagle's medium/F12 (DMEM/F12 Glutamax, Gibco) supplemented with N2-supplement, glucose (0.6\%), HEPES $(5 \mathrm{mM})$, human serum albumin $(0.5 \%)$, heparin $(0.0002 \%)$, non-essential amino acid solution and penicillin-streptomycin ( $100 \mathrm{units} / \mathrm{ml})$. On day 3 , cells were cultured in neural induction media, consisting in the neural basal media supplemented with retinoic acid (500 nM), dibutyryl cAMP (1 mM) and growth factors like BDNF $(10 \mathrm{ng} / \mathrm{ml}$; Peprotech), GDNF $(10 \mathrm{ng} / \mathrm{ml}$; Peprotech) and IGF-1 (10 ng/ml; R\&D systems). We perform time-lapse phase-contrast microscopy within the first $24 \mathrm{~h}$ after neural induction media was added directly to the cells.

Immunocytochemistry. A standard immunocytochemical protocol was used as previously described ${ }^{25,62}$. Cells were plated onto collagen IV coated plastic or glass coverslips, and maintained in basal media or neural induction media. Cells were rinsed with PBS and fixed in freshly prepared paraformaldehyde (4\%). Fixed cells were blocked for $2 \mathrm{~h}$ in PBS containing normal horse serum (10\%) and Triton X-100 (0.25\%) and incubated overnight at $4{ }^{\circ} \mathrm{C}$ with antibodies against $\beta$-III-tubulin (TUJ1; 1:500, Covance) in PBS containing normal horse serum (1\%) and Triton X-100 (0.25\%). On the next day, cells were rinsed and incubated with the secondary antibody: Alexa Fluor 594 (anti-mouse; 1:500, Molecular Probes). Cell nuclei were counterstained with DAPI $\left(0.2 \mathrm{mg} / \mathrm{ml}\right.$ in PBS, Molecular Probes). Alexa Fluor $488^{\circ}$ phalloidin (Molecular Probes) was used to selectively stains F-actin. Data are representative of ten independent experiments per condition.

Images and data analyses. Analyses and photography of visible and fluorescent stained samples were carried out as previously described ${ }^{25}$. We used an inverted Leica DM IRB microscope equipped with a digital camera Leica DFC350FX (Nussloch) and confocal laser scanning microscope Leica TCS-SP8. Digitized images 
were analyzed using LASX Leica confocal software. We used Photoshop software to improve the visibility of fluorescence images without altering the underlying data.

Received: 5 February 2021; Accepted: 27 May 2021

Published online: 08 June 2021

\section{References}

1. Waddington, C. H. The Strategy of the Genes. A Discussion of Some Aspects of Theoretical Biology (Allen \& Unwin, Crows Nest, 1957).

2. Wang, J., Zhang, K., Xu, L. \& Wang, E. Quantifying the Waddington landscape and biological paths for development and differentiation. Proc. Natl. Acad. Sci. 108, 8257-8262 (2011).

3. Venkei, G. Z. \& Yamashita, Y. M. Emerging mechanisms of asymmetric stem cell division. J. Cell Biol. 217, 3785-3795 (2018).

4. Rajagopal, J. \& Stanger, B. Z. Plasticity in the adult: how should the waddington diagram be applied to regenerating tissues?. Dev. Cell 36, 133-137 (2015).

5. Raff, M. Adult stem cell plasticity: Fact or artifact?. Annu. Rev. Cell Dev. Biol. 19, 1-22 (2003).

6. Tata, P. R. \& Rajagopal, J. Cellular plasticity: 1712 to the present day. Curr. Opin. Cell Biol. 43, 46-54 (2016).

7. Jopling, C., Boue, S. \& Izpisua-Belmonte, J. C. Dedifferentiation, transdifferentiation and reprogramming: Three routes to regeneration. Nat. Rev. Mol. Cell Biol. 12, 79-89 (2011).

8. Merrell, A. J. \& Stanger, B. Z. Adult cell plasticity in vivo: de-differentiation and transdifferentiation are back in style. Nat. Rev. Mol. Cell Biol. 17, 413-425 (2016).

9. Mills, J. C., Stanger, B. Z. \& Sander, M. Nomenclature for cellular plasticity: Are the terms as plastic as the cells themselves?. EMBO J https://doi.org/10.15252/embj.2019103148 (2019).

10. Reid, A. \& Tursun, B. Transdifferentiation: Do transition states lie on the path of development?. Curr. Opin. Syst. Biol. 11, 18-23 (2018).

11. Black, I. B. \& Woodbury, D. Adult rat and human bone marrow stromal stem cells differentiate into neurons. Blood Cells Mol. Dis. 27, 632-636 (2001).

12. Woodbury, D., Reynolds, K. \& Black, I. B. Adult bone marrow stromal stem cells express germline, ectodermal, endodermal, and mesodermal genes prior to neurogenesis. J. Neurosci. Res. 69, 908-917 (2002).

13. Munoz-Elias, G., Woodbury, D. \& Black, I. B. Marrow stromal cells, mitosis and neuronal differentiation: Stem cell and precursor functions. Stem Cells 21, 437-448 (2003).

14. Jeong, J. A. et al. Rapid neural differentiation of human cord blood-derived mesenchymal stem cells. NeuroReport 15, 1731-1734 (2004).

15. Suon, S. et al. Transient differentiation of adult human bone marrow cells into neuron-like cells in culture: Development of morphological and biochemical traits is mediated by different molecular mechanisms. Stem Cells Dev. 13, 625-635 (2004).

16. Ning, H., Lin, G., Lue, T. F. \& Lin, C. S. Neuron-like differentiation of adipose tissue-derived stromal cells and vascular smooth muscle cells. Differentiation 74, 510-518 (2016).

17. Azedi, F. et al. Comparative capability of menstrual blood versus bone marrow derived stem cells in neural differentiation. Mol. Biol. Rep. 44, 169-182 (2017).

18. Radhakrishnan, S. et al. In vitro transdifferentiation of human adipose tissue-derived stem cells to neural lineage cells-A stagespecific incidence. Adipocyte 8, 164-177 (2019).

19. Hernández, R. et al. Differentiation of human mesenchymal stem cells towards neuronal lineage: clinical trials in nervous system disorders. Biomol. Ther. 28, 34-44 (2020).

20. Choudhary, P., Gupta, A. \& Singh, S. Therapeutic advancement in neuronal transdifferentiation of mesenchymal stromal cells for neurological disorders. J. Mol. Neurosci. https://doi.org/10.1007/s12031-020-01714-5 (2020).

21. Krabbe, C., Zimmer, J. \& Meyer, M. Neural transdifferentiation of mesenchymal stem cells-A critical review. APMIS 113, 831-844 (2005).

22. Bertani, N., Malatesta, P., Volpi, G., Sonego, P. \& Perris, R. Neurogenic potential of human mesenchymal stem cells revisited: analysis by immunostaining, time-lapse video and microarray. J. Cell Sci. 118, 3925-3936 (2005).

23. Neuhuber, B. et al. Reevaluation of in vitro differentiation protocols for bone marrow stromal cells: Disruption of actin cytoskeleton induces rapid morphological changes and mimics neuronal phenotype. J. Neurosci. Res. 77, 192-204 (2004).

24. Lu, P., Blesch, A. \& Tuszynski, M. H. Induction of bone marrow stromal cells to neurons: Differentiation, transdifferentiation, or artifact?. J. Neurosci. Res. 77, 174-191 (2004).

25. Bueno, C., Martínez-Morga, M. \& Martínez, S. Non-proliferative neurogenesis in human periodontal ligament stem cells. Sci. Rep. https://doi.org/10.1038/s41598-019-54745-3 (2019).

26. Castro-Malaspina, H. et al. Characterization of human bone marrow fibroblast colony-forming cells (CFU-F) and their progeny. Blood 56, 289-301 (1980).

27. Samsonraj, R. M. et al. Concise review: multifaceted characterization of human mesenchymal stem cells for use in regenerative medicine. Stem Cells Transl. Med. 6, 2173-2185 (2017).

28. Gronthos, S., Mankani, M., Brahim, J., Robey, P. G. \& Shi, S. Postnatal human dental pulp stem cells (DPSCs) in vitro and in vivo. Proc. Natl. Acad. Sci. 97, 13625-13630 (2000).

29. Liu, H., Gronthos, S. \& Shi, S. Dental pulp stem cells. Methods Enzymol. 419, 99-113 (2006).

30. Arthur, A., Rychkov, G., Shi, S., Koblar, S. A. \& Gronthos, S. Adult human dental pulp stem cells differentiate toward functionally active neurons under appropriate environmental cues. Stem Cells 26, 1787-17895 (2008).

31. Mohan, S. P. \& Ramalingam, M. Dental pulp stem cells in neuroregeneration. J. Pharm. Bioallied Sci. https://doi.org/10.4103/jpbs. JPBS_229_20 (2020).

32. Foudah, D. et al. Expression of neural markers by undifferentiated mesenchymal-like stem cells from different sources. J. Immunol. Res. 2014, 987678. https://doi.org/10.1155/2014/987678 (2014).

33. Bueno, C. \& Martínez, S. Neurogenesis similarities in different human adult stem cells. Neural Regen. Res. 16, 123-124 (2021).

34. Erharter, A., Rizzi, S., Mertens, J. \& Edenhofer, F. Take the shortcut-direct conversion of somatic cells into induced neural stem cells and their biomedical applications. FEBS Lett. 593, 3353-3369 (2019).

35. Skinner, B. M. \& Johnson, E. E. Nuclear morphologies: their diversity and functional relevance. Chromosoma 126, 195-212 (2017).

36. Olins, D. E. \& Olins, A. L. Nuclear envelope-limited chromatin sheets (ELCS) and heterochromatin higher order structure. Chromosoma 118, 537-548 (2009).

37. Fenech, M. et al. Molecular mechanisms of micronucleus, nucleoplasmic bridge and nuclear bud formation in mammalian and human cells. Mutagenesis 26, 125-132 (2011).

38. Guo, X. et al. The molecular origins and pathophysiological consequences of micronuclei: New insights into an age-old problem. Mutat. Res. 779, 1-35 (2019). 
39. Yokoi, A. et al. Mechanisms of nuclear content loading to exosomes. Sci. Adv. https://doi.org/10.1126/sciadvaax8849 (2019).

40. Guerrero-Cázares, H. et al. Cytoarchitecture of the lateral ganglionic eminence and rostral extension of the lateral ventricle in the human fetal brain. J. Comp. Neurol. 519, 1165-1180 (2011).

41. Doetsch, F., Garcia-verdugo, J. M. \& Alvarez-buylla, A. Cellular composition and three-dimensional organization of the subventricular germinal zone in the adult mammalian brain. J. Neurosci. 17, 5046-5061 (1997).

42. Capilla-Gonzalez, V., Cebrian-Silla, A., Guerrero-Cazares, H., Garcia-Verdugo, J. M. \& Quiñones-Hinojosa, A. Age-related changes in astrocytic and ependymal cells of the subventricular zone. Glia 62, 790-803 (2014).

43. Cebrián-Silla, A. et al. Unique organization of the nuclear envelope in the post-natal quiescent neural stem cells. Stem Cell Rep. 9 , 203-216 (2017).

44. Wittmann, M. et al. Synaptic activity induces dramatic changes in the geometry of the cell nucleus: interplay between nuclear structure, histone H3 phosphorylation, and nuclear calcium signaling. J. Neurosci. 29, 14687-14700 (2009).

45. Bond, A. M., Ming, G. L. \& Song, H. Adult mammalian neural stem cells and neurogenesis: five decades later. Cell Stem Cell 17, 385-395 (2015).

46. Lazutkin, A., Podgorny, O. \& Enikolopov, G. Modes of division and differentiation of neural stem cells. Behav. Brain Res. https:// doi.org/10.1016/j.bbr.2019.112118 (2019).

47. Doetsch, F., Garcia-verdugo, J. M. \& Alvarez-buylla, A. Cellular composition and three-dimensional organization of the subventricular germinal zone in the adult mammalian brain. J. Neurosci. 17, 5046-5061 (1997).

48. Doetsch, F., Garcia-verdugo, J. M. \& Alvarez-buylla, A. Regeneration of a germinal layer in the adult mammalian brain. Proc. Natl. Acad. Sci. 96, 11619-11624 (1999).

49. Doetsch, F., Caillé, I., Garcia-verdugo, J. M. \& Alvarez-buylla, A. Subventricular zone astrocytes are neural stem cells in the adult mammalian brain. Cell 97, 703-716 (1999).

50. Seri, B., Garcia-verdugo, J. M., McEwen, B. S. \& Alvarez-buylla, A. Astrocytes give rise to new neurons in the adult mammalian hippocampus. J. Neurosci. 21, 7153-7160 (2001).

51. Filippov, V. et al. Subpopulation of nestin-expressing progenitor cells in the adult murine hippocampus shows electrophysiological and morphological characteristics of astrocytes. Mol. Cell Neurosci. 23, 373-382 (2003).

52. Kronenberg, G. et al. Subpopulations of proliferating cells of the adult hippocampus respond differently to physiologic neurogenic stimuli. J. Comp. Neurol. 467, 455-463 (2003).

53. Seri, B., Garcia-verdugo, J. M., Collado-Morente, L., McEwen, B. S. \& Alvarez-buylla, A. Cell types, lineage, and architecture of the germinal zone in the adult dentate gyrus. J. Comp. Neurol. 474, 359-378 (2004).

54. Kempermann, G., Jessberger, S., Steiner, B. \& Kronenberg, G. Milestones of neuronal development in the adult hippocampus. Trends Neurosci. 27, 447-452 (2004).

55. Li, G. \& Hidalgo, A. Adult neurogenesis in the drosophila brain: the evidence and the void. Int. J. Mol. Sci. 18, 1. https://doi.org/ $10.3390 /$ ijms $21186653(2020)$.

56. Rakic, P. Adult neurogenesis in mammals: An identity crisis. J. Neurosci. 22, 614-618 (2002)

57. Cooper-Kuhn, C. M. \& Kuhn, H. G. Is it all DNA repair? Methodological considerations for detecting neurogenesis in the adult brain. Brain Res. Dev. 134, 13-21 (2002).

58. Breunig, J. J., Arellano, J. I., Macklis, J. D. \& Rakic, P. Everything that glitters isn't gold: a critical review of postnatal neural precursor analyses. Cell Stem Cell 1, 612-627 (2007).

59. Kuhn, H. G., Eisch, A. J., Spalding, K. \& Peterson, D. A. Detection and phenotypic characterization of adult neurogenesis. Cold Spring Harb. Perspect. Biol. 8, a025981. https://doi.org/10.1101/cshperspect.a025981 (2016).

60. Sorrells, S. F. et al. Positive controls in adults and children support that very few, if any, new neurons are born in the adult human hippocampus. J. Neurosci. 41, 2554-2565 (2021).

61. Blau, H. M., Brazelton, T. H. \& Weimann, J. M. The evolving concept of a stem cell: entity or function?. Cell 105, 829-841 (2001).

62. Bueno, C. et al. Human adult periodontal ligament-derived cells integrate and differentiate after implantation into the adult mammalian brain. Cell Transplant. 22, 2017-2028 (2013).

\section{Author contributions}

C.B. conceived of the study, designed the study, carried out the molecular lab work and drafted the manuscript. M.M. and D.G. carried out the molecular lab work and participated in data analysis. J.M. helped draft the manuscript. S.M. conceived of the study, helped draft the manuscript and financial support. All authors reviewed the manuscript.

\section{Funding}

This work has been funded by Instituto de Salud Carlos III through the project "RD16/001/0010" (Co-funded by European Regional Development Fund/European Social Fund "Investing in your future") and Spanish MINECO/ AEI/FEDER (SAF2017-83702-R).

\section{Competing interests}

The authors declare no competing interests.

\section{Additional information}

Supplementary Information The online version contains supplementary material available at https://doi.org/ 10.1038/s41598-021-91566-9.

Correspondence and requests for materials should be addressed to C.B.

Reprints and permissions information is available at www.nature.com/reprints.

Publisher's note Springer Nature remains neutral with regard to jurisdictional claims in published maps and institutional affiliations. 
(c) (i) Open Access This article is licensed under a Creative Commons Attribution 4.0 International cc) License, which permits use, sharing, adaptation, distribution and reproduction in any medium or format, as long as you give appropriate credit to the original author(s) and the source, provide a link to the Creative Commons licence, and indicate if changes were made. The images or other third party material in this article are included in the article's Creative Commons licence, unless indicated otherwise in a credit line to the material. If material is not included in the article's Creative Commons licence and your intended use is not permitted by statutory regulation or exceeds the permitted use, you will need to obtain permission directly from the copyright holder. To view a copy of this licence, visit http://creativecommons.org/licenses/by/4.0/.

(C) The Author(s) 2021 